\title{
Annual Scientific Meeting, Edmonton, September 27-30, 2007
}

\section{Rho Kinase Underpins Airway Smooth Muscle Hyperreactivity in Naive Caveolin-1 Knockout Mice}

S. Martin, S. Basu, D. Schaafsma, A.J. Halayko, Department of Physiology, Faculty of Medicine, University of Manitoba and Manitoba Institute of Child Health, Winnipeg, MB

Caveolin-1 (Cav-1) can modulate intracellular signaling pathways in airway smooth muscle (ASM) that may mediate inflammation, contraction, and/or proliferation. Previously, we observed enhanced methacholine (MCh)induced ASM contraction ex vivo and enhanced airway resistance in vivo in mice lacking Cav-1. In the present study, we investigated the possible role of Rho kinase, protein kinase $\mathrm{C}(\mathrm{PKC})$, and $\mathrm{p} 42 / \mathrm{p} 44$ mitogen-activated protein kinase (MAPK) in the enhanced MCh-induced ASM contraction and airway resistance in Cav-1 knockout mice (Cav-1 KO). Tracheal rings from naive, 8-week-old, female Cav-1 KO and genetically matched B6129SF2/J mice were isolated and mounted on a wire myograph. Isometric contraction in response to $\mathrm{MCh}$ was measured in the presence or absence of selective inhibitors of Rho kinase (Y-27632, $1 \mu \mathrm{M}$ and $10 \mu \mathrm{M})$, PKC (bisindolylmaleimide, $3 \mu \mathrm{M}$ ), and p42/p44 MAPK (U0126, $3 \mu \mathrm{M}$ ). The role of Rho kinase in MCh-induced airway resistance (Raw) was also investigated in vivo using a Scireq ventilator. Thirty minutes prior to measuring respiratory mechanics, Cav-1 KO and B6129SF2/J mice were exposed to aerosolized saline or Rho kinase inhibitor (5 mM Y27632, 4 minutes). Using excised tracheal rings, maximum MCh-induced contraction was increased significantly in preparations from Cav-1 $\mathrm{KO}(8.93 \pm 0.77 \mathrm{mN})$ compared to $\mathrm{B} 6129 \mathrm{SF} 2 / \mathrm{J}$ mice $(6.45 \pm 0.64 \mathrm{mN})$. Pretreatment with $10 \mu \mathrm{M}$ Y-27632 reduced sensitivity and maximum response to $\mathrm{MCh}$ in both tissues and normalized the difference in contractile responses between mouse strains. Notably, whereas tracheas from Cav-1 KO exhibited concentration-dependent responses to Y-27632, maximum suppression was achieved with $1 \mu \mathrm{M}$ of inhibitor in B6129SF2/J mice. Though we did observe a modest effect in suppressing $\mathrm{MCh}$-induced contractile force with bisindolylmaleimide and U0126 treatment, the effect was of equal magnitude on Cav-1 KO and B6129SF2/J mice, suggesting that the contribution of PKC and $\mathrm{p} 42 / \mathrm{p} 44$ MAPK to contractile responses is not changed in Cav-1 $\mathrm{KO}$ mice. As we previously observed in vivo, Cav-1 KO mice exhibited a significant increase in Raw and tissue resistance $(\mathrm{G})$. However, consistent with our ex vivo experiments, inhaled Y-27632 both decreased Raw and G and normalized these parameters between mouse strains. Collectively, the data suggest that Cav-1 modulates the contribution of Rho kinase in MCh-mediated ASM contraction, which is a principal determinant of Raw.

\section{Flow Cytometry Analysis of Neutrophil CD62L Shedding for Rapid Diagnosis of IRAK-4 Deficiency: Utility and Caveats in Comparison to Cytokine Responses}

Andrew C. Issekutz, Derek Rowter, Christine Riddell, TongJun Lin, Departments of Pediatrics, MicrobiologyImmunology, and Pathology, Dalhousie University, Halifax, NS

We evaluated a recently reported screening test for IRAK-4 deficiency based on the downregulation of CD62L (L-selectin) on blood neutrophils (PMN) upon Toll-like receptor (TLR) agonist stimulation with two known IRAK-4-deficient patients and a newborn sibling. From control donors and the carriers, $>70 \%$ of PMNs shed CD62L after 60-minute stimulation of blood with bacterial endotoxin (LPS; TLR4 agonist), lipopeptides (FSL-1 or Pam $_{3}$ Cys-SK4; TLR2 agonists), and R848 (Resiquimod; TLR7/8 agonist). With PMN of IRAK-4-deficient patients, CD62L shedding with LPS was virtually absent $(<15 \%)$, and there was no shedding with lipopeptides or R848. In contrast, the PMN of a newborn sibling at age 7 days had an intermediate shedding response to LPS (50\% shed), although there was no shedding after stimulation with FSL and only $20 \%$ shedding with R848. However, at 7 weeks of age, response to LPS became virtually nil $(<15 \%$ CD62L shedding) and there was no response to FSL or R848. All 
patients' PMNs had a normal shedding response to $S$. aureus peptidoglycan (PGN) (TLR2 and other receptors). The IRAK-4-deficient patients did not mount an IL-6 or a TNF- $\alpha$ response to LPS, R848, or PGN in whole blood. The 7-day-old sibling had a small IL-6 response to LPS (5.5-fold increase) and a normal response to PGN. At 7 weeks of age, there was no IL- 6 or TNF- $\alpha$ response to LPS, R848, or lipopeptides, but a diminished response to PGN was still present. Genotyping confirmed that the newborn carried the same two IRAK-4 gene mutations (C144G; 631delG) as the affected sibling, each mutation causing a premature stop codon. Thus, CD62 analysis by flow cytometry on blood PMN following stimulation with TLR agonists is a valuable rapid screen for IRAK-4 deficiency, but LPS and PGN are not reliable stimuli to detect IRAK-4 deficiency. The TLR2 lipopeptide agonists FSL or $\mathrm{Pam}_{3}$ Cys-SK4 and the TLR7/8 agonist R848 should be included along with LPS as agonists. PGN is of no value in IRAK-4 deficiency screening. Research funded by the Canadian Institutes of Health Research (CIHR).

\section{An Environmental Exposure Chamber (EEC)- Specific Rhinoconjunctivitis Quality of Life Questionnaire: The Symptoms of Seasonal Allergic Rhinitis Correlate with the Quality of Life (QOL) of Patients with Ragweed Allergy in the EEC}

A.M. Salapatek, P. Patel, C. Shah, K. Fischer von Weikersthal-Drachenberg, J. Amersdorffer, Allied Research International, Mississauga, ON; Allergy Therapeutics plc, Worthing, UK; AllerPharma Inc., Toronto, ON

Background: Recognizing the need to assess QOL, we developed an EEC-specific rhinoconjunctivitis QOL (EECRQOL) questionnaire to measure the QOL of patients. The questionnaire contains four domains: Nonnose/Eye (NE), Practical Problems (PP), Emotional (E), Global Assessment (GA). Aims: To examine the relationship between QOL and Total Symptom Scores (TSS) of subjects during pollen exposure in the EEC toward validation of the EEC-RQOL questionnaire. Methods: Ragweed-sensitive subjects were exposed to ragweed pollen $(3,500 \pm$ grains/ $\mathrm{m}^{3}$ ) in the EEC for 3 hours in which they recorded instantaneous TSS on 4 consecutive days (Baseline Visits $2-5)$. Subjects were given four weekly injections of Pollinex Quattro Ragweed (PQ, 300, 700, 2,000, 6,000 SU, $n=87$ ), and 3 weeks later, they were assessed at the EEC on 4 consecutive days (visits 11-14). The correlation between EEC-RQOL and TSS was analyzed with Pearson's correlation $(p<.01$ significant). The correlation between EECRQOL scores and the TSS visit 5 (when TSS were maximized) and for visit 113 weeks post-treatment were analyzed. Results: Each individual domain of the questionnaire and the total QOL score significantly correlated with TSS at baseline (PP, $r=.53$; NE, $r=.31$; E, $r=.31$; GA, $r=.33$; and Total, $r=.39, p<.01)$ and after PQ treatment (PP, $r=.80$; NE, $r=.62$; E, $r=.58$; GA, $r=$ .50 ; and Total, $r=.74, p<.001)$. The correlations between TSS and EEC-RQOL for total and all individual domains improved after treatment compared to pretreatment values and data clustered where symptoms were low and EEC-RQOL was high. Conclusions: This first study toward the validation of the EEC-RQOL Questionnaire shows significant, positive correlations between SAR symptoms and QOL of subjects in the EEC and thus suggests cross-sectional construct validity of the questionnaire. Increased correlation values and data shifts after PQ treatment indicate that improvements in symptoms were accompanied by subject EEC-RQOL improvement, demonstrating that the EEC-RQOL questionnaire is a useful tool in evaluating QOL of subjects in the EEC. Funding: This study was supported by AllerPharma Inc., Toronto, ON, and Allergy Therapeutics plc, Worthing, UK. This abstract was submitted to a meeting of the EAACI in 2007.

\section{Evaluation of the Safety and Immunogenicity of Pollinex Quattro Grass (PQ) in Patients Suffering from Grass and Rye Allergies}

P. Patel, A.M. Salapatek, C. Shah, P. Tanna, D. Iyer, K. Fischer von Weikersthal Drachenberg, J. Amersdorffer, Allied Research International, Mississauga, ON; AllerPharma, Mississauga, ON; Allergy Therapeutics plc, Worthing, UK

$\mathrm{PQ}$ is an allergy vaccine designed to enhance beneficial immune responses and improve safety with an allergoid to reduce $\operatorname{IgE}$ reactivity and retain $\operatorname{IgG}$ stimulation and allergoid adsorption onto a L-tyrosine depot to slow bioavailability and minimize adverse reactions. Aims: To compare the immunogenicity and safety of 3 PQ allergoid dose regimens to placebo. Methods: A double-blind, placebo-controlled study including 74 patients allergic to grass/rye pollen was conducted. After a screening visit (V1), patients received four weekly injections at V2-V5 with one of three PQ test dose regimens: therapeutic (300, $800,2,000,2,000 \mathrm{SU} ; n=22)$, intermediate $(150,300,800$, $800 \mathrm{SU} ; n=23$ ), low ( 4 doses $150 \mathrm{SU} ; n=19$ ), or placebo $(n=10)$. V6 occurred 2 weeks post-treatment. Blood samples were taken at V1, V3, V4, V5, and V6. ANOVA was used to compare the change over baseline in serum concentration of grass-specific immunoglobulins (IgG, 
IgG1, IgG4 to Timothy/Rye/June grasses) between the three PQ regimens and placebo. Safety was assessed by adverse events (AEs). Results: Primary analyses of Timothy Grass-specific immunoglobulins showed significant changes over baseline occurred most often with the therapeutic dose by V6, with net changes over placebo in specific IgG, IgG1, IgG4 of 59.9\%, 87.8\%, 105.9\% ( $p=$ .042 .005, .009), respectively. The intermediate dose had fewer significant changes in immunoglobulin levels from baseline compared to placebo. The low-dose group was ineffective in enhancing immunoglobulin levels. Similar trends in immunoglobulins specific to Rye and June grass were observed. Forty-nine patients (66\%) experienced drug-related AEs that were mostly mild or moderate in severity, related to injection-site conditions. There were no severe AEs, deaths, or severe systemic AEs. Conclusions: Increasing doses of PQ Grass allergoid increased PQ Grass immunogenicity in a dose-dependent manner, with the highest dose conferring significant changes in immunoglobulin levels, and was safe and well tolerated at all dosing regimens. Study supported by AllerPharma Inc., Toronto, ON, and Allergy Therapeutics plc., Worthing, UK. This abstract was presented in part at the ACAAI 2006.

\section{Assessment of the Residual Allergenicity of Pollinex Quattro Ragweed Using Skin-Prick Testing}

P. Patel, A.M. Salapatek, C. Shah, P. Tanna, M. Chudak, K. Fischer von Weikersthal Drachenberg, J. Amersdorffer, Allied Research International, Mississauga, ON; AllerPharma, Mississauga, ON; Allergy Therapeutics plc, Worthing, UK

Background: Pollinex Quattro (PQ) Ragweed employs an allergoid adsorbed onto L-tyrosine depot to reduce allergenicity. Objectives: To compare the relative residual allergenicities of unmodified native ragweed allergen to the allergoid alone or in combination with the depot with or without adjuvant and their relative safety and tolerability. Methods: A single-blind study with 12 patients allergic to ragweed pollen was conducted. After a screening, patients had SPTs with the following test products: native ragweed allergen $(1.0909 \% \mathrm{w} / \mathrm{v}+6$ serial 1:3 dilutions), ragweed allergoid $(1.0909 \% \mathrm{w} / \mathrm{v})$, ragweed allergoid tyrosine adsorbed $(6,000 \mathrm{SU} / 0.5 \mathrm{~mL})$, ragweed allergoid tyrosine adsorbed plus monophosphoryl lipid A (MPL) $(6,000 \mathrm{SU} /$ $0.5 \mathrm{~mL})$, positive control histamine solution $(0.1 \%)$, and negative control glycerinated extraction medium (GEM). SPTs for each test product were duplicated on each forearm. Residual allergenicity of test products was determined by the difference in the area $\left(\mathrm{mm}^{2}\right)$ of the wheal response for each test product and GEM. The seven wheal areas from the native allergen were plotted against concentration to produce a concentration-response plot. The wheal areas from the three allergoid products were compared to that plot and the corresponding native allergen concentrations were estimated using linear interpolation. Patients remained for 6-hour late-phase assessment. Safety was assessed from adverse event (AE) reports. Results: The calculated median activity of the aqueous allergoid $(1.0909 \% \mathrm{w} / \mathrm{v})$ was equivalent to approximately $1 / 47$ th the corresponding aqueous native allergen. The calculated median activity of PQ ragweed was approximately $1 / 225$ th of aqueous native allergen. No drug-related AEs or late-phase allergic reactions $>10 \mathrm{~cm}$ were observed following exposure to any allergens tested. Conclusions: The results indicate that the allergoid contained in PQ ragweed elicits only a fraction of the allergenicity of its progenitor product, at the same concentration of pollen; as well, PQ ragweed was safe and well tolerated in this study. Funding: This study was supported by AllerPharma Inc., Toronto, ON, and Allergy Therapeutics plc, Worthing, UK. This abstract was presented in part at the ACAAI 2006.

\section{The Safety and Clinical Efficacy of Pollinex Quattro Ragweed Assessed in an Environmental Exposure Chamber (EEC)}

P. Patel, A.M. Salapatek, C. Shah, P. Tanna, E. Kreiner, K. Fischer von Weikersthal-Drachenberg, J. Amersdorffer, Allied Research International, Mississauga, ON; AllerPharma, Toronto, ON; Allergy Therapeutics plc, Worthing, UK

Pollinex Quattro (PQ) Ragweed is a new, ultra-shortcourse AV with three advances. Use of an allergoid adsorbed onto an L-tyrosine depot reduces IgE reactivity, improving safety. Use of an adjuvant monophosphoryl lipid A (MPL) positively immunomodulates allergoid activities to enhance AV efficacy reducing PQ treatment to four preseasonal injections. Aims: To evaluate the efficacy and safety of PQ in an EEC, PQ efficacy was assessed by Total Symptom Scores (TSS, nasal + nonnasal), immune responses, and an EEC-specific rhinoconjunctivitis quality of life questionnaire (RQLQ). PQ safety was assessed by adverse event (AE) report. Methods: A double-blind, placebo-controlled study including 177 ragweed-sensitive patients was performed. After screening, patients were studied in the EEC in 3-hour ragweed exposures on 4 consecutive days for baselines. Patients were given four weekly injections with either PQ $(300,700$, 2,000, $6000 \mathrm{SU}, n=87)$ or placebo $(n=90)$. Three weeks after the last injection, EEC assessments were repeated. ANOVA was used to compare PQ to placebo for TSS, IgG, 
IgE, and RQLQ. Safety was assessed by AE reports. Results: Post-treatment, the reduction in TSS over baseline with PQ was significantly larger $(-6.61)$ than with placebo $(-4.47)(p=.007)$. PQ increased ragweed-specific IgG significantly more than placebo, 3,247.2 vs $36.6 \mathrm{ng} / \mathrm{mL}(p$ $<.001)$. There was no significant difference in the $\operatorname{IgE}$ levels between PQ and placebo. RQLQ indicated that the PQ group had greater improvement in practical problems and global assessments compared to placebo. One hundred fifty-three patients had AEs that were mostly mild or moderate in severity and related to the injection site. There were no severe AEs, deaths, or severe systemic AEs. Conclusions: PQ treatment results in significant symptom relief, progressing from "moderate" to "mild." PQ increases specific IgG with no safety issues. These findings likely contribute to real changes in patient quality of life and indicate PQ ragweed effectiveness. Funding: Study supported by AllerPharma Inc., Toronto, ON, and Allergy Therapeutics plc, Worthing, UK. This abstract was presented at the ACAAI 2006.

\section{Increased IgG Levels Induced by Pollinex Quattro Ragweed (PQ) in Ragweed-Allergic Patients Studied in an Environmental Exposure Chamber (EEC) Are Maintained during Follow-Up in the Natural Ragweed Pollen Season}

P. Patel, A.M. Salapatek, C. Shah, S. McCue, K. Fischer von Weikersthal-Drachenberg, J. Amersdorffer, Allied Research International, Mississauga, ON; Allergy Therapeutics plc, Worthing, UK; AllerPharma Inc., Toronto, ON

Background: PQ is designed to enhance beneficial immune responses with an allergoid to reduce $\operatorname{IgE}$ reactivity but retain IgG stimulatory action. Aims: To examine serum IgG/IgE after treatment with PQ during ragweed exposure in an EEC and during follow-up in natural ragweed season in southern Ontario. Methods: A randomized, double-blind, placebo-controlled study to evaluate ragweed-specific IgG and IgE in ragweed-allergic patients treated with PQ $(n=90)$ compared to placebo $(n$ $=87$ ). The treatment study group was primed on visits V2-V5, was treated with PQ, and 3 weeks after treatment was exposed to ragweed allergen during four daily visits (V11-V14) in the EEC. A follow-up study examined a subset of these patients who completed the treatment study (PQ: $n=44$; placebo: $n=52$ ) throughout the subsequent ragweed season. The follow-up study consisted of seven visits (F1-F7) occurring 14 days apart over a 12week period, with F1 coinciding with V14 of the treatment study. Immunoglobulin testing was performed at EEC visits V5, V11, and V14, and follow-up visits F5 and F7. Results: Mean IgG levels were greater for the PQ group (V5 [baseline]: 583.6; V11: 3,809.7; V14: 3,834.6 ng/mL) compared to the placebo group (V5 [baseline]: 1,114.1; V11: 1,157.0; V14: $1,150.7 \mathrm{ng} / \mathrm{mL})(p<.001)$ at all visits in the EEC. IgG levels remained significantly higher during the follow-up visits for PQ versus placebo for all visits (F5: $2,215.5$ vs $1,470.2$; F7: $1,884.0$ vs $1,432.3$ ) ( $p<.001$ ). The ragweed-specific IgE levels between $\mathrm{PQ}$ and placebo were not significant at any visit, although mean IgE levels were lower with PQ in the follow-up study compared to placebo (F5: 30.1 vs 34.9; F7: 27.0 vs 30.6). Conclusions: A significant increase in IgG was shown for PQ versus placebo-treated patients at all time points in the EEC study. IgG levels remained elevated for 16 weeks after PQ treatment and throughout the natural ragweed season. Funding: Study supported by AllerPharma Inc., Toronto, ON, and Allergy Therapeutics plc, Worthing, UK. Abstract originally presented at EAACI 2007.

\section{Tree Pollen Allergoids in Pollinex Quattro Tree Immunotherapy Reduce the Residual Allergenicity as Assessed with Skin-Prick Tests (SPTs)}

P. Patel, A.M. Salapatek, C. Shah, M. Chudak, K. Jethwa, K. Fischer von Weikersthal-Drachenberg, J. Amersdorffer, Allied Research International, Mississauga, ON; Allergy Therapeutics plc, Worthing, UK; Allerpharma Inc., Toronto, ON

Rationale: The Pollinex Quattro (PQ) Tree employs modified allergens (allergoids) adsorbed onto L-tyrosine depot to reduce allergenicity. Relative residual allergenicities of unmodified native tree allergen to the allergoid alone or in combination with the depot with or without adjuvant (monophosphoryl lipid A) were assessed with SPTs. Methods: A single-blind study with 12 birch, hazel, and alder pollen-allergic patients was conducted. Patients had SPTs with the following test products: native tree allergen $(1.25 \% \mathrm{w} / \mathrm{v}+6$ serial $1: 3$ dilutions $)$, tree allergoid $(1.25 \% \mathrm{w} / \mathrm{v})$, tree allergoid tyrosine adsorbed (4,000 SU/ $0.5 \mathrm{~mL})$, tree allergoid tyrosine adsorbed plus adjuvant (4,000 SU/0.5 mL, PQ tree), positive control histamine solution $(0.1 \%)$, and negative control glycerinated extraction medium (GEM). Residual allergenicity of each test product was determined by the difference in area of their wheal response and GEM. The seven wheal areas from the native allergen were plotted against concentration. The wheal areas from the three allergoid products were compared to that plot and the corresponding native allergen concentrations estimated using linear interpola- 
tion. Patients had 6-hour late-phase assessments. Safety was determined from adverse event (AE) reports. Results: The calculated median activity of the aqueous allergoid $(1.25 \% \mathrm{w} / \mathrm{v})$ was equivalent to $1 / 26$ th the corresponding aqueous native allergen. The calculated median activity of PQ tree was approximately $1 / 300$ th of aqueous native allergen. No drug-related AEs and no late-phase allergic reactions $>10 \mathrm{~cm}$ were observed following any allergen exposures. Conclusions: The allergoid contained in the PQ Tree elicits only a fraction of the allergenicity of its progenitor product, at the same concentration of pollen, and the PQ Tree was safe and well tolerated in this study. Funding: Study supported by Allerpharma Inc., Toronto, ON, and Allergy Therapeutics plc, Worthing, UK. Abstract originally presented at the AAAAI 2007.

\section{The Availability of the Epinephrine Auto-Injector in Children with Peanut Allergy}

Moshe Ben-Shoshan, Rhoda Kagan, Marie-Noël Primeau, Reza Alizadehfar, Nina Verreault, Joyce W. Yu, Nathalie Nicolas, Lawrence Joseph, Elizabeth Turnbull, Claire Dufresne, Yvan St. Pierre, Ann Clarke, Divisions of Pediatric Allergy and Clinical Immunology, Clinical Epidemiology, and Allergy and Clinical Immunology, McGill University Health Centre, Montreal, QC; Division of Allergy and Clinical Immunology, North York General Hospital, Toronto, ON; Department of Epidemiology and Biostatistics, McGill University, Montreal, QC; Association Québecoise des Allergies Alimentaires

Background: Peanut allergy represents a major health problem and is receiving increasing attention in the medical literature. Avoidance of peanut is often difficult, and the principal treatment of an acute allergic reaction is prompt administration of epinephrine. Objective: We sought to describe the availability of epinephrine auto-injectors and determine factors that might affect their availability in peanut-allergic children living in Quebec. Methods: Two hundred seventy-two children with peanut allergy were queried on their epinephrine auto-injector. Logistic regressions were used with the Bayes Information Criteria to select the best predictive factors associated with the availability of the epinephrine auto-injector. Results: Four of 272 children were not prescribed epinephrine auto-injectors, although they were diagnosed as peanut allergic; $48.1 \%$ (95\% CI $42-$ 54.3) of children did not carry the epinephrine auto-injector on them while at school. Epinephrine auto-injectors were initially prescribed by allergists in 52.6\% (95\% CI 46.4-58.7) of children and in $29.9 \%$ (95\% CI 24.4-35.7) of children upon last renewal, respectively. Among those 7 years or older, those who experienced a severe reaction were more likely to carry their epinephrine auto-injector with them (odds ratio 3.3; 95\% CI 1.35-8.3). Conclusion: Almost 50\% of peanut-allergic children might experience a delay in the treatment of anaphylaxis as a result of limited access to their device. Another factor that might be associated with less than optimal anaphylactic treatment was a relatively low rate of prescriptions administered by the allergist, the health care provider most likely to educate on the potential risk for anaphylaxis and on the appropriate use of the auto-injector.

\section{Development and Implementation of an Evidence- Based Care Map for the Management of Children Admitted with Asthma to Winnipeg Children's Hospital}

L. Galloway, C. Gillespie, C. Cronin, W. Watson, H. Pasterkamp, J. Carson, C. Madrid, A. Studney, T. Mortimer, M. Hanna, J. Bullard, A. Dixon, S. Al-Harbi, S. Hutton, K. Valeri, A. Esquivel, B. Sproll, Child Health Program, Winnipeg Regional Health Authority, Winnipeg, MB

Asthma is a common reason for admission at our children's hospital. A 2003 clinical audit suggested that there were opportunities for care improvements. The main project objective was to develop and pilot an evidencebased care map for management of children $>2$ years admitted for asthma. The Care Map was developed by a multidisciplinary group using Project Methodology. Development included review of existing asthma literature and care provided at Children's Hospital; creation of the Care Map and supporting documents, including a clinical scoring tool; gaining approval from various programs and committees; and education of targeted health care professionals. The Care Map was piloted on one medical ward at Children's Hospital. Implementation began in June 2005 and included daily communication and support to staff, joint problem solving, mini chart audits, and regular feedback. Evaluation included chart audits for children admitted for asthma between June 1, 2005, and March 31, 2006, and for those admitted in September 2006. Results included a decrease in length of stay, an increase in the use of spirometry, earlier transition to the use of salbutamol by metered dose inhaler, and improvements in discharge planning and teaching. Conclusions include the following:

- A belief that the Asthma Clinical Scoring Tool has facilitated more efficient weaning of inhalation treatments

- Support from the Child Health leadership, commitment of working group members, and a project management approach were critical success factors 
- Consistency and efficiency of care for this group of children have improved at our hospital

\section{Physicians' Perspectives of Allergic Rhinitis (AR) in Canada}

S. Waserman, R.R. Schellenberg, P.K. Keith, M. Desrosiers, Department of Medicine, University of British Columbia, Vancouver, BC; Department of Medicine, McMaster University, Hamilton, ON; Department of Medicine, McGill University, University of Montreal, Montreal, QC

Rationale: To evaluate physicians' perspectives of the burden of AR and effectiveness of therapy. Methods: Physicians were selected through random screening of a national physician database to participate in a structured telephone interview in July 2006. Included were 100 general practitioners (GPs), 30 allergists, and 30 otolaryngologists (ENTs). Results: Physicians reported that $>$ $90 \%$ of AR patients have bothersome symptoms, the worst being nasal congestion. They identified asthma and sinusitis as comorbid conditions of most concern and estimated that 31 to $41 \%$ of AR patients have asthma and 24 to $32 \%$ have sinusitis. Allergy skin testing was performed always by $80 \%$ of allergists, $17 \%$ of ENTs, and $8 \%$ of GPs. Sixty-two percent of physicians demonstrated use of nasal sprays when prescribed, but $17 \%$ of GPs did so only when asked versus $3 \%$ of specialists. Onethird of allergists and one-tenth of ENTs named the ARIA guidelines without prompting. All cited a need for allergy CME (85-90\%) and better patient education. Twenty percent of GPs and 38\% of patients believed there were no truly effective therapies for AR versus $0 \%$ of allergists and $3 \%$ of ENTs yet felt that frequent AR symptoms could be prevented in most cases (100\% allergists, 90\% ENTs, $83 \%$ GPs vs $64 \%$ patients). Physicians estimated that one-third of patients stop taking their medication during therapy, mainly due to lack of efficacy rather than side effects. Conclusions: Although physicians recognize the burden of AR on patients, there remains a need for better education of both physicians and patients about AR in addition to better therapies.

\section{Allergic Sensitization to Cockroach Allergens Is PAR-2 Dependent}

Narcy Arizmendi, Melanie Abel, Cory Ebeling, Harissios Vliagoftis, Pulmonary Research Group, University of Alberta, Edmonton, $A B$

Introduction: A number of common aeroallergens have serine protease activity, which is important for allergic sensitization. Cockroach allergens are very common in urban environments and are associated with increases in the incidence and severity of asthma. Cockroach extracts can mediate some of their effects through the proteaseactivated receptor 2 (PAR-2). PAR-2 is activated by serine proteases, including some aeroallergens, and has been implicated in inflammatory reactions. Furthermore, we have shown that activation of this receptor leads to allergic sensitization to concomitantly administered antigens. To study the role of PAR-2 in sensitization to common allergens we developed a murine model using cockroach extract as allergen. Hypothesis: Cockroach extract, administered intranasally (i.n.) in mice, induces allergic sensitization characterized by inflammation and airway hyperresponsiveness (AHR) through the activation of PAR-2 on airway epithelium and/or lung dendritic cells. Methods: For allergic sensitization, cockroach extract was administered i.n. to mice daily for 5 days. Mice were later challenged with cockroach extract for another 4 consecutive days and then were assessed for AHR and allergic airway inflammation (AI). To study the role of PAR-2 in allergic sensitization, mice were administered an anti-PAR2-blocking antibody i.n. before each cockroach administration during the sensitization phase. Results: Mice that were sensitized and challenged with cockroach showed eosinophilic inflammation and AHR. Administration of the PAR-2-blocking antibody completely inhibited the development of AHR and AI. Cockroach extract administration led to altered dendritic cell migration to lymph nodes and dendritic cell maturation. Conclusions: Cockroach extract induces PAR-2-dependent allergic airway sensitization when given i.n. in mice. This model will allow us to investigate the mechanisms of allergic sensitization to allergens with serine protease activity.

\section{Monomeric IgE Induces Mast Cell Activation In Vivo in the Absence of Specific Antigen}

Melanie Abel, Harissios Vliagoftis, Pulmonary Research Group, University of Alberta, Edmonton, $A B$

Introduction: IgE has been shown to induce mast cell survival, proliferation, and cytokine production in the absence of antigen-induced Fc?RI cross-linking through low-level spontaneous receptor dimerization. Mast cellfibroblast interactions induce the release of proteases important for tissue remodeling, such as MMP-9. This suggests that the atopic state, characterized by high $\operatorname{IgE}$ levels, may be sufficient to induce changes leading to airway remodeling and inflammation before the development of manifestations of asthma or other allergic diseases. 
Hypothesis: IgE-induced mast cell activation in vivo induces the release of mediators involved in tissue remodeling, such as MMP-9, in the absence of specific antigen. Methods: To study whether IgE could upregulate MMP-9 release in vivo in the absence of allergen, we injected monomeric $\operatorname{IgE}(25 \mu \mathrm{g})$ into one ear pinna of naive mice. The same volume of saline was administered to the other ear as control. The ears were removed 24 hours later for histologic analysis or homogenized and analyzed by zymography for the presence of MMP-9. Results: Administration of $\operatorname{IgE}$ without antigen increased the MMP-9 content of the ear by 2 -fold compared to the ears receiving saline. Saline did not change the ear MMP-9 content compared to non-injected ears. Conclusions: The MMP-9 content of the mouse ear was increased following administration of IgE, indicating the initiation of mast cell-dependent inflammatory and remodeling pathways in the absence of relevant antigen. Interactions between monomeric IgE and mast cells play an important role in initiating tissue remodeling and are important in understanding the development of allergic inflammation and asthma.

\section{Complementary and Alternative Medicine Use in an Adult Asthma Program}

Jody Yue, Adam Romanovsky, Dilini Vethanayagam, Department of Medicine, Faculty of Medicine and Dentistry, University of Alberta

Background: Asthma is a chronic inflammatory disease of the airways affecting 9 to $10 \%$ of adult Canadians. Complementary and alternative medicine (CAM) is a term used in reference to nontraditional medical (allopathic medicine) or nonmedicinal therapies that may include breathing techniques, herbal medication use, acupuncture, homeopathy, nutritional therapies, chiropractics, and mind body therapy. CAM use has been noted to occur in the majority of the general population. Objective: To determine the prevalence of CAM use in adult asthmatics referred to a single asthma subspecialist clinic. Methods: Following approval by the University of Alberta Health Research Ethics Board, adult asthma patient charts were reviewed and entered into a database to investigate the prevalence and reasons for use of CAM therapy at the time of initial encounter of patients in the clinic. A total of 51 patient charts between the years of 2003 and 2006 were assessed. Results: From the 51 charts reviewed, 19 (37\%) patients had used CAM either for asthma or nonasthma reasons. There was a larger proportion of male patients (40\%) who used CAM compared to females (36\%).
Common reasons for CAM therapy included treatment of asthma symptoms (16\%), allergies (16\%), upper respiratory tract infections (21\%), and musculoskeletal problems $(63 \%)$. In this population, those born in North America reported the highest proportion of CAM use (45\%) as opposed to those born elsewhere. Conclusions: Although CAM use is prevalent in the general population, this was seen less frequently than would have been expected in this referral population of asthmatics. It may be that patients who choose to attend adult asthma subspecialty clinics may be less likely to use nonallopathic, physician-prescribed treatments as opposed to other asthmatics.

\section{Exam Stress Does Not Cause Change in Lung Function and Quality of Life in "Healthy Asthmatics"}

Dilini Vethanayagam, Nicholas Coupland, Dean Befus, Harissios Vliagoftis, Pulmonary Research Group and Department of Psychiatry, Faculty of Medicine and Dentistry, University of Alberta

Background: Over $30 \%$ of asthmatics experience exacerbations of their asthma during periods of stress. However, few studies have addressed the pathogenetic mechanisms of this effect. Mild asthmatics demonstrate increased airway inflammation after allergen challenge during high stress periods. In that study the patients were not selected for stress-induced asthma and no effects on asthma control were shown. Objective: To examine differences in lung function and quality of life and recruitment of immune cells into the airways of mild asthmatics during low- (V1) and high-stress (V2) periods. Methods: Adult asthmatics were recruited following approval by the University of Alberta Health Research Ethics Board. No financial compensation was provided. Subjects who participated attended the lab for a screening visit during which we obtained their informed consent followed by a brief clinical exam, spirometry, methacholine challenge, allergen skin tests, sputum induction, and Mini International Neuropsychiatirc Interview (MINI). Eligible individuals had their low-stress visit (V1) at least 14 days prior to examinations and the high-stress visit (V2) 24 hours beforehand. Spirometry, methacholine challenge, and induced sputum were performed during both V1 and V2, along with collection of blood and urine samples and administration of four questionnaires: general (EuroQol5D) and disease-specific Asthma Quality of Life Questionnaires (AQLQ), Mood and Anxiety Syndrome Questionnaire (MASQ), and Perceived Stress Scale (PSS). 
Results: Nineteen subjects were screened in detail. Five subjects failed the MINI screen. Nine subjects dropped out due to the time commitment required for study participation. Five subjects (three female, two male) were able to complete the study; four of these were atopic (one nonatopic male). Four subjects were on low-dose inhaled steroids during the study period. Two of the five subjects had stress-related worsening of their asthma by history. Low-stress (V1) and high-stress (V2) visits were compared. Subjects who completed the study showed no change in PSS, although a trend toward an increase at V2 was noted. Similarly, no change was noted in MASQ. No significant change was noted in lung function. No significant change was noted in general (EQ-5 D) or disease-specific AQLQ evaluations between low- and high-stress visits, although a significant difference was noted in urinary cortisol levels (reduction noted during high stress). Conclusion: Underlying psychiatric diagnoses (DSM-4) were prevalent in this apparently "healthy asthmatic" population recruited for the above study and resulted in the exclusion of close to half the subjects who would have otherwise been interested in participation. In the remaining subjects from whom significant psychiatric comorbidity was excluded, no significant worsening of asthma control was noted, although these individuals did not have significant stress with examinations as assessed by the MASQ and PSS scales. Funding: This study was supported by a grant from the Alberta MSI Foundation.

\section{Lung Epithelial Integrins as Pattern-Recognition Receptors: Implications for Innate Immunity and Inflammation}

Sean K. Gravelle, Rebecca J. Barnes, Marina Ulanova, Northern Ontario School of Medicine, Lakehead University, Thunder Bay, ON

Epithelial cells (ECs) are currently recognized as primary elements generating inflammatory signals to activate other cells in the lung. Although the importance of ECs for innate immunity is established, the underlying mechanisms are incompletely understood. We have previously found that $\beta_{1}$ integrins in lung ECs provide costimulatory signals regulating inflammatory responses (Ulanova et al. Am J Physiol 2005;288:L497-507). Importantly, epithelial integrins and their ligands are involved in adhesion and internalization of several human pathogens. The aim of the present study is to test the hypothesis that lung EC integrins serve as receptors to recognize pathogen-associated molecules and mediate the innate immune response to the opportunistic pathogen
Pseudomonas aeruginosa. To determine molecular mechanisms of integrin involvement in innate immunity, we used an in vitro model of $P$. aeruginosa infection of A549 cells. To investigate interactions of bacteria with ECs, $P$. aeruginosa strain PAK was chromosomally labeled with a green fluorescent protein gene using a mini-Tn7 delivery system. Using several fluorescence-based detection systems, we established that the natural $\alpha_{5} \beta_{1}$ integrin ligand fibronectin mediates bacterial adhesion to ECs. $P$. aeruginosa infection caused rapid transcriptional upregulation of $\alpha_{5}$ and $\beta_{4}$ integrins followed by the increased cell surface protein expression. The surface expression of $\alpha_{5}$ and $\beta_{1}$ integrins increased shortly following bacterial exposure without alterations of mRNA expression, suggesting protein redistribution within the cells. Interestingly, killed $P$. aeruginosa did not alter integrin expression, demonstrating the importance of live bacteriacell interactions. The data indicate that $P$. aeruginosa are capable to modulate integrin gene/protein expression in lung ECs, potentially using fibronectin to alleviate bacterial binding to $\alpha_{5} \beta_{1}$ integrins. Upon their engagement, integrin receptors can initiate intracellular signaling involved in innate immune and inflammatory responses to the pathogen. Lung epithelial integrins may represent important therapeutic targets in pulmonary infection caused by P. aeruginosa. Support: NSERC.

\section{Association of Dystrophin Glycoprotein Complex (DGC) with Human Airway Smooth Muscle Maturation}

Pawan Sharma, Gerald Stelmack, Karol McNeill, Helmut Unruh, Andrew J. Halayko, Departments of Physiology and Internal Medicine, Section of Respiratory Disease, National Training Program in Allergy and Asthma, and Section of Thoracic Surgery, University of Manitoba, Winnipeg, MB; Biology of Breathing Group, Manitoba Institute of Child Health, Winnipeg, $M B$

Airway smooth muscle (ASM) cells contribute to asthma pathogenesis through their capacity to switch between a synthetic/proliferative and contractile phenotype. The multimeric dystrophin glycoprotein complex (DGC) spans the sarcolemma, providing a mechanical link between the intracellular actin cytoskeleton and extracellular matrix, and it serves as a scaffold for intracellular signaling proteins. Loss of DGC subunits is associated with myopathies such as Duchene muscular dystrophy (DMD) in humans. The DGC is abundant and organized into linear plasma membrane arrays in contractile smooth muscle cells. The functional role of DGC in human ASM 
and whether its expression is a unique feature of mature contractile human airway smooth muscle is not fully known. We tested the hypothesis that maturation to a contractile phenotype is associated with increased accumulation of DGC in human ASM cells. Protein lysates were obtained from subconfluent, serum-fed cultures (synthetic/proliferative phenotype) and from confluent cultures subjected to prolonged serum deprivation (contractile phenotype). Effects of phosphatidylinositide-3kinase (PI3K) inhibitors and laminin-competing peptide, both of which are required for phenotype maturation, on DGC subunit abundance were measured. Western blotting confirmed that the abundance of $\beta$-dystroglycan; $\beta$-, $\delta$-, and ?-sarcoglycan; and dystrophin increased 6- to 8-fold in 4-day serum-deprived human ASM cultures; concomitant accumulation of smooth muscle myosin (smMHC) and calponin, established markers of the contractile phenotype, was also induced with 4 days of serum deprivation. Notably, laminin-competing peptide (YIGSR, $1 \mu \mathrm{m}$ ) and PI3K inhibitors (LY-294002, $20 \mu \mathrm{m}$, or wortmannin, 100 $\mathrm{nm}$ ) abrogated myocyte maturation and the accumulation of both DGC components and contractile apparatusassociated proteins. Moreover, immunocytochemistry showed that the accumulation of DGC subunits is localized to cells that exhibit positive staining for smMHC. These studies demonstrate that the accumulation of DGC is an integral feature of the process of phenotype maturation of human ASM cells. Our results indicate the DGC is a reliable marker for contractile human ASM cells in vitro. The functional significance of the increased accumulation of DGC in a mature contractile cell needs further investigation to better understand the physiology of smooth muscle in diseases like asthma. This project is supported by grants from CIHR, the Canada Research Chair Program, and Manitoba Institute of Child Health $(\mathrm{MICH})$. P.S. holds a studentship from $\mathrm{MICH}$ and the National Training Program in Allergy and Asthma. A.J.H. hold a Canada Research Chair in Airway Cell and Molecular Biology.

\section{Nitric Oxide Regulates Mast Cell Function by Altering Cellular Fructose 1,6 Bisphosphate Levels upon Nitration of Aldolase}

Yokananth Sekar, A. Dean Befus, Pulmonary Research Group, Department of Medicine, University of Alberta, Edmonton, $A B$

Mast cells (MCs) are primary effector cells of IgEmediated allergic inflammation. MC-derived nitric oxide (NO), as well as exogenous NO, regulates MC activities.
We hypothesized that protein tyrosine nitration, a posttranslational modification mediated by NO, plays a regulatory role in MCs. Using a hypothesis-generating proteomic approach, we identified an enzyme in the glycolytic pathway, aldolase, as a target for nitration in MC. Nitrated proteins of HMC-1, a human mast cell line, were assessed using two-dimensional electrophoresis and Western blot with antinitrotyrosine antibody. Mass spectrometry was used to characterize proteins selectively nitrated upon treating the cells with SNOG, a NO donor. Treatment with $500 \mu \mathrm{M}$ of SNOG for 4 hours selectively nitrates tyrosine residues at positions 3 and 59 of aldolase A in HMC-1 cells. This nitration was associated with reduced aldolase enzymatic activity and corresponding increase in its substrate, fructose 1,6 bisphosphate (FBP), intracellularly in HMC-1. Since FBPs have been reported to inhibit MC degranulation in animal models, we studied its effect on MC function using an in vitro IL-8 assay. Exogenous FBP treatment results in a dose-dependent reduction of IL-8 production of HMC-1. This is the first report of tyrosine nitration in human aldolase and also in MCs. Preliminary experiments with LAD-2, a mature human MC line, and human cord blood-derived MCs also revealed aldolase nitration upon NO treatment, thereby favouring aldolase as a potential target in NO-mediated control of MC function. Aldolase nitration has the potential to regulate $\mathrm{MC}$ function through multiple mechanisms, including elevated FBP levels. FBP may act through enzymes like PLC $\gamma$ and PLD2 or IP3, an intracellular messenger. Analyses of the possible links between aldolase nitration, altering FBP levels, and the regulation of MC function may help identify novel therapeutic targets to treat allergic diseases. This work was funded by the Canadian Institutes of Health Research and Alberta Lung Association.

\section{Cyclin-Dependent Kinase 5 Regulates Eosinophil Degranulation via a Calpain-Dependent Pathway}

S.O. Odemuyiwa, D.J. Adamko, F. Davoine, C. Wu, C. Majaesic, R. Moqbel, Department of Medicine, and Paediatrics, Pulmonary Research Group, University of Alberta, Edmonton, $A B$

Introduction: Eosinophils may contribute to allergic airway inflammation through the release of stored granule mediators and reactive oxygen species. The intracellular mechanisms governing the release of these mediators are poorly understood. Recent studies have suggested that cyclin-dependent kinase 5 (cdk5) may be important in the process of granule exocytosis in neurons, insulin-produ- 
cing cells, and neutrophils. Objectives: To determine the expression of cdk5, and cdk5 activators (p35 and p39), and its role in eosinophil mediator release. Methods: Western blotting, RT-PCR, and flow cytometry were used to determine the expression of cdk5, p35, and p39 in eosinophils obtained from atopic human donors. Following treatment with roscovitine, a specific inhibitor of cdk5, the release of eosinophil peroxidase (EPO) was measured in cells activated with secretory IgA-coated beads. In addition, the effect of roscovitine and calpeptin, a calpain inhibitor, on the adhesion of eosinophils to fibroncetin-coated plates was measured. Following extraction of total phosphorylated proteins, cellular moieties associated with cdk5-mediated exocytosis were identified. Results: We detected cdk5 and its activators, p35 and p39, in peripheral blood eosinophils. Eosinophil cdk5 was shown to have functional kinase activity and express Munc 18c, a cdk5 substrate that directly regulates granule fusion. Roscovitine, calpeptin, and a pool of specific silencing RNA (siRNA) the release of eosinophil peroxidise following activation. Adhesion to fibronectin was also inhibited by roscovitine and calpain. Conclusions: Cdk5 is an important intracellular regulator of eosinophil adhesion to fibronectin and EPO secretion. Funding: Canadian Institutes of Health Research (CIHR) and Alberta Heritage Foundation for Medical Research (AHFMR).

\section{Regulation of Secretion of Anti-Inflammatory Prohormone SMR1 by Autonomic Stimulation in Rat Submandibular Glands}

Katherine Morris, Paul Forsythe, Sam Harirforoosh, Ryan Hoeve, Ron Mathison, A. Dean Befus, Pulmonary Research Group, Department of Medicine, University of Alberta, Edmonton, AB; McMaster University, Hamilton, ON; University of Calgary, Calgary, $A B$

Stress-induced activation of the sympathetic nervous system modifies endocrine functions of salivary glands, thus systemically regulating allergic inflammation. In rats, a cleavage product of the prohormone SMR1 (submandibular rat 1) is produced in the submandibular gland and acts systemically to decrease allergic pulmonary inflammation and anaphylaxis. A mimic of the smallest active fragment of this product, the D-isomeric tripeptide feG, is being developed as a therapeutic and is effective in rats, mice, dogs, sheep, cats, and isolated human neutrophils. It has shown efficacy in animal models of pulmonary inflammation, food allergy, septic shock, pancreatitis, and spinal cord injury. Pharmaceutical development will be aided by information on the endogenous regulation of SMR1 and related antiinflammatory peptides in neuroendocrine pathways. We have evaluated the effect of sympathetic and parasympathetic mimetics on the expression, processing, and secretion of SMR1 in rats. SMR1 is present in rat submandibular glands in at least 52 species that result in part from $\mathrm{N}$-glycosylation and cleavage of the protein. Beta-adrenergic (sympathomimetic) stimulation causes the rapid disappearance of SMR1 protein from the submandibular gland and appearance of the protein in saliva and plasma. Cholinergic (parasympathomimetic) stimulation causes secretion of SMR1 into saliva without significantly depleting the protein from the gland. The release of SMR1 and its fragments into saliva and plasma in response to stress may be important in regulating the response to allergic inflammation. Future work will aim to evaluate the role of this stress-regulated salivary peptide release in models of endotoxic shock and asthma.

\section{A Valved Holding Chamber (VHC) Manufactured from Electrostatic Charge-Dissipative Materials Affords Superior Delivery of Medication Compared with Nonconducting Devices If Inhalation Is Delayed}

H. Harkness, J.P. Mitchell, M.W. Nagel, Trudell Medical International, London, $\mathrm{ON}$

VHCs are often prescribed for patients who have difficulty coordinating the timing of inhalation with actuation of their pressurized metered dose inhaler (pMDI). Particle deposition caused by electrostatic effects can reduce performance under these circumstances. We report a study in which delivery of Ventolin-HFA via a group of VHCs ( $n=5$ devices, AeroChamber Max, Trudell Medical International, London ON) manufactured from charge-dissipative materials was compared with performance of a representative VHC manufactured from nonconducting polymer (OptiChamber Advantage, Respironics Inc., Cedar Grove, NJ). The AeroChamber Max VHCs were evaluated immediately after removal from their packaging. The OptiChamber Advantage VHCs were washed in mild detergent, rinsed, and drip-dried before use following manufacturer instructions. A proprietary apparatus that interfaced between the VHC mouthpiece and induction port leading to an eight-stage Andersen cascade impactor was used to simulate 2- and 5-second delay intervals between pMDI actuation and the onset of sampling at $28.3 \mathrm{~L} / \mathrm{min}$. Values of fine particle mass (FPM 
( $\mu \mathrm{g} /$ actuation $)$ ), based on particles $<4.7 \mu \mathrm{m}$ aerodynamic diameter for 2-second delay, were $23.8 \pm 4.8 \mu \mathrm{g}$ and $6.7 \pm$ $2.3 \mu \mathrm{g}$ for the AeroChamber Max and OptiChamber Advantage VHCs, respectively. The corresponding values for 5 -second delay were $19.1 \pm 2.1 \mu \mathrm{g}$ and $2.3 \pm 1.3 \mu \mathrm{g}$. FPM for the pMDI alone (no delay) was $28.5 \pm 2.5 \mu \mathrm{g}$. The AeroChamber Max VHCs significantly outperformed the OptiChamber Advantage VHC group, even after prewashing, to mitigate electrostatic charge (unpaired $t$ test at each delay interval, $p<.001)$. This study indicates the potential for significant adverse clinical implications for uncoordinated patients using a nonconducting VHC, who may delay inhalation.

\section{Immunotherapy for Hymenoptera Allergy}

Ian MacDougall, McMaster University, Hamilton, ON

Over the past number of years immunotherapy for Hymenoptera allergy has become an extremely effective treatment option for patients. However, this treatment strategy requires that these patients are accurately identified through both their clinical history and appropriate diagnostic tests. Recently, much has been written about the diagnostic dilemma of patients who have a convincing clinical history of venom allergy but subsequently have a negative venom skin test. It is of great clinical importance to properly identify these patients as they may still require immunology to help prevent potentially life-threatening reactions. We present a chart review of a subset of 50 patients in the greater Hamilton area who were referred to the allergy service for evaluation of Hymenoptera allergy. Their clinical histories were analyzed to determine the likelihood that they experience a true allergic reaction to a venom sting. Specific criteria for symptoms included shortness of breath, systemic hives, and throat swelling, among others. It was then determined if these patients had a negative or positive skinprick testing to a selection of Hymenoptera extracts and finally the results of in vitro RAST testing if ordered. The goal is to identify the prevalence of patients in the Hamilton area who have a convincing clinical history and positive RAST test despite having a negative skin-prick test. Future components of this study will involve sending bloodwork for RAST results to other centres as well as Hamilton's testing site to determine if the sensitivities of these tests differ.

\section{The Role of Rac2 in Eosinophil Superoxide Release and Allergic Airway Responses}

Andrea N. Lo, Troy Mitchell, Melanie Abel, James Dooley, Harissios Vliagoftis, David A. Williams, Marc E. Rothenberg,
Gary Eitzen, Nives Zimmermann, Paige Lacy, Pulmonary Research Group, Department of Medicine, and Department of Cell Biology, University of Alberta, Edmonton, AB; Division of Experimental Hematology and Division of Allergy and Immunology, Cincinnati Children's Hospital Medical Center, University of Cincinnati College of Medicine, Cincinnati, $\mathrm{OH}$

Background: Superoxide production from eosinophils undergoing respiratory burst correlates with asthma severity and is thought to contribute to allergic symptoms by causing edema and tissue inflammation. Superoxide generation is dependent on activation of NADPH oxidase by a GTP-bound Rho-related guanosine triphosphatase (GTPase), Rac1 or its homolog Rac2. While neutrophils express mainly Rac2, and Rac2 is the dominant protein that activates NADPH oxidase, it is not known whether Rac1 or Rac2 preferentially activates the oxidase in eosinophils. Our earlier studies (Fulkerson et al. Blood 2005;106:436) indicated that Rac2 is required for eotaxin2-induced chemotaxis in eosinophils, demonstrating functional consequences in eosinophils. Here we determined whether Rac2 is a central regulator of mediator release and immune function in eosinophils. Objectives: To determine whether Rac2 regulates the production of superoxide release from eosinophils and whether Rac2 mediates inflammation and airway hyperresponsiveness (AHR). Methods: We isolated splenic eosinophils from CD2-IL-5 transgenic mice (WT) and Rac2-deficient mice bred against the CD2-IL-5 transgenic background (Rac2 $\mathrm{KO} / \mathrm{IL}-5 \mathrm{Tg}$ ) and compared their ability to release superoxide in response to phorbol myristate acetate (PMA). To determine allergic inflammatory responses, we subjected mice to intraperitoneal sensitization with ovalbumin (OVA) and alum followed by intranasal OVA challenge or intranasal sensitization to cockroach allergens and compared the responses of WT C57Bl/6 mice with Rac2 KO mice. Responses were determined by bronchoalveolar lavage cell counts and Penh measurements for AHR. Results: Whole spleen and MACS-purified splenic eosinophils from Rac2 KO/IL-5 Tg mice showed a reduction of superoxide release in comparison to WT mice. This was similar to Rac2 $\mathrm{KO}$ neutrophils, which exhibit a deficiency ( $\approx 30 \%$ of control values) in superoxide release. In both models of airway inflammation and AHR, Rac2 KO mice developed eosinophilia in BAL samples and hyperresponsiveness that was similar to control wild-type mice. Conclusions: These findings suggest that gene deletion of Rac2 affects eosinophil superoxide release but not its transmigration into the airways. This is in contrast to the model of neutrophil-mediated acute lung 
injury in Rac2 $\mathrm{KO}$ mice, in which there is significantly less airway inflammation and injury. We propose that while Rac2 is important for superoxide release in both neutrophils and eosinophils, Rac1 may instead be a critical regulator of eosinophil migration.

\section{Perioperative Anaphylaxis: The Halifax Experience}

L. Connors, G. Lacuesta, Dalhousie University, Halifax, NS

Background: Anaphylaxis is a clinical diagnosis based on history, physical, and appropriate investigations. Perioperative anaphylaxis has been described to a variety of agents used in induction and maintenance of anesthesia, as well as antibiotics, latex, and various dyes. The most commonly described agents for perioperative anaphylaxis are neuromuscular blocking agents. In particular, the amine-steroid agents such as rocuronium and pancuronium are more commonly implicated as the culprit agent in intraoperative anaphylaxis. The incidence of intraoperative anaphylaxis has been documented as quite variable, with rates between 1 in 5,000 and 1 in 20,000 surgical cases. The goal of this series was to describe the various cases seen in Halifax, Nova Scotia. Methods: Records from an adult allergy and clinical immunology office were reviewed from 2002 to 2006 to identify patients seen with a possible diagnosis of perioperative anaphylaxis. Nine cases are presented, including clinical course and investigations, to identify the culprit agent. Results: The cases reviewed revealed that seven of nine cases were female patients. The cases ranged in age between 18 and 76 years old. The site of surgery was genitourinary in four of nine cases and head and neck in two of nine cases, with one case each of abdomen, breast, and back surgery. Only two of nine patients had a documented history of atopy or asthma. Of note, only one patient was on a betablocker preoperatively. In this case series rocuronium was identified as the culprit agent in four of nine cases. Penicillin was implicated in three cases, whereas cephazolin and vancomycin were implicated in one case each. In one case investigations did not reveal an obvious cause for the reaction. Conclusion: The findings from this case series are comparable to the trends seen in the literature in this area. Rocuronium was the most commonly implicated agent, followed by antibiotics. Also, perioperative anaphylaxis has been described to occur more in female patients and, in particular, in those undergoing genitourinary procedures, as was seen in this case series. Further research and standardization of testing in this area are required.

\section{Differential Activation Patterns of RAB27A in Human Eosinophils and Cell Lines: Interdonor Variability}

Jason J. Coughlin, Redwan Moqbel, Pulmonary Research Group, Department of Medicine, University of Alberta, Edmonton, AB

Background: Eosinophils play an effector role in airway damage in asthma and related disorders since their secreted granule-stored products can induce many of the clinical features of asthma. Degranulation of eosinophils requires the activity of several GTP-binding proteins, many of which remain unidentified. We hypothesized that Rab27A is one of these GTP-binding proteins because, in other cells, it interacts with effectors that regulate vesicle motility, docking, and fusion. Subjects with Rab27A deficiency develop Griscelli syndrome, which is characterized by immunodeficiency and partial albinism due to secretory defects in various cell types. Objectives: To assess Rab27A activation in human eosinophil degranulation. Methods: RT-PCR and Western blotting were performed on human peripheral blood eosinophils and the eosinophil-like cell lines AML14.3D10 and HL-60 clone 15. To assess Rab27A activation, we developed a novel assay allowing the pulldown of the active, GTP-bound form. Results: Rab27A was expressed in human peripheral blood eosinophils and transiently activated by secretory IgA stimulation. The kinetics of Rab27A activation varied between donors. High eosinophilic donors ( $>25 \times 10^{6}$ cells/100 mL of blood) displayed a more rapid cycle of Rab27A activation and inactivation as compared to eosinophils from other donors ( $<15 \times 10^{6}$ cells/100 mL of blood). Rab27A was also expressed in the HL-60 clone 15 "eosinophil-like" cell line but was not expressed in AML14.3D10 cells. In the HL-60 clone 15 cells, Rab27A was also activated by sIgA stimulation; however, the kinetics of activation were much slower than that of peripheral blood eosinophils. Rab27A was also activated by IFN- $\gamma$ and eotaxin in these cells. Conclusions: Rab27A is activated in human eosinophils following sIgA stimulation, suggesting that it may induce secretory granule movement and exocytosis in these cells. The differential activation pattern of Rab27A provides a pattern of the differences between resting and activated eosinophils. Funding: Canadian Institutes of Health Research and the Alberta Heritage Foundation for Medical Research.

\section{Interlukin-10-Treated Human Dendritic Cells Inhibit Th2 Responses of Atopic Subjects and Induce the Differentiation of Regulatory T Cells}

Xiuling Li, Xiaobei Zhang, Jennifer Town, Beth Davis, Don Cockcroft, John R. Gordon, Immunology Research Group, 
University of Saskatchewan; Division of Respiratory Medicine, Royal University Hospital, Saskatoon, SK

Background: Allergic asthma is a chronic disease characterized by Th2 inflammation. We previously demonstrated that IL-10-treated dendritic cells (DCs) can abrogate asthmatic responses in mice, including airway hyperresponsiveness (AHR), eosinophilia, Th2 cytokine secretion, and serum IgE/IgG1. Herein we investigated the ability of analogous IL-10-treated human DC to similarly tolerize Th2 responses of atopic subjects $\mathrm{T}$ cells. Methods: Purified $\mathrm{CD}^{+}{ }^{+} \mathrm{T}$ cells from atopic donors were cultured with autologous allergen-pulsed IL-10treated human monocyte-derived DCs ( $\left.{ }^{\text {all }} \mathrm{DC}_{\mathrm{IL}-10}\right)$, either alone or together with autologous immunostimulatory DCs in order to assess the tolerogenic activity of the ${ }^{\text {all }} \mathrm{DC}_{\mathrm{IL}-10}$. In addition, the abilities of putative regulatory $\mathrm{T}$ cells arising from these cultures to inhibit Th2 responses of autologous $\mathrm{T}$ cells were assessed. Outcome measures included $\mathrm{T}$ cell proliferation and IL-4, IL-5, and IL-13 secretion (assessed by $\left[{ }^{3} \mathrm{H}\right]$-thymidine incorporation and ELISA, respectively) and characterization of the induced Treg by qRT-PCR for FoxP3, LAG3, CTLA4, and IL-10 mRNA expression. Results: We found that IL-10 fully inhibited human DC maturation, as determined by the reduced expression of costimulatory molecules (CD80, CD86), stimulatory cytokines (IL-6, IL-12), and improvement in phagocytic activity. When cultured alone with autologous Th2 cells, these ${ }^{\text {all }} \mathrm{DC}_{\mathrm{IL}-10}$ induced antigenspecific $\mathrm{T}$ cell anergy, and when added to cocultures of immunostimulatory DC-activated Th2 cells, they also inhibited Th2 cell proliferation and cytokine expression. We report also that a population of LAG $3^{+} \mathrm{CTLA} 4^{+}$, IL-10secreting regulatory $\mathrm{T}$ cells arose in these cocultures and that these induced Treg could, in turn, effectively suppress autologous activated Th2 cell proliferation and cytokine secretion in a contact-dependent manner. Conclusion: These data indicate that IL-10-treated human DCs can tolerize allergic immune response by driving the differentiation of regulatory $\mathrm{T}$ cells and suggest that this strategy could potentially be exploited as a therapeutic strategy for allergic disease.

\section{Establishment of an Asthma Education Centre in the Community: A Pilot Project}

N. Toma ${ }^{1}$, C. Sharpe, T.H. Liem, C.A. Gillespie, A.B. Becker, J.J. Liem, Windsor Allergy Asthma Education Centre, Windsor, ON; Children's Asthma Education Centre, Winnipeg, $M B$
Rationale: The Children's Asthma Education Centre (CAEC), based in Winnipeg, has shown that asthma education can decrease health care utilization by children for acute asthma and improve quality of life. The Windsor Allergy Asthma Education Centre (WAAEC) was established in July 2006 with close ties to CAEC with an objective of providing a similar service in the Windsor/ Essex County Region in Ontario. Objective: To determine the feasibility of establishing an asthma education centre in the community setting and to describe our 1-year experience. Methods: WAAEC began to educate patients with asthma in September 2006. Initial evaluation of the program is based on surveys completed by participants 2 weeks post-education sessions. Results: Two hundred twelve patients ( 66 children/parents and 146 adults) were educated from September 2006 to June 2007. Thirty participants (9 children, 21 adults) had been hospitalized for asthma prior to the education session. Forty-nine participants ( 4 children, 45 adults) had been diagnosed with asthma $>10$ years and had never received formal asthma education. Of the returned evaluations, over $90 \%$ of participants found that the education sessions met their expectations and increased their knowledge and understanding of asthma. Forty-four percent of evaluations found education regarding medications, their uses, and proper device technique to be the most important item they learned. Conclusion: The WAAEC's first year was a success in providing asthma education to patients in Windsor and Essex County. Further evaluation will need to examine long-term benefits of asthma education and the feasibility of operating an asthma education centre in a nonhospital and nonuniversity setting. Future evaluation will include questionnaires (Juniper Quality of Life, Asthma Control, CAEC Asthma Quiz) at regular intervals (3 months, 6 months, and 1 year).

\section{Clinical Course and Immunological Features of Siblings with IRAK-4 Deficiency}

Elana Lavine, Chaim M. Roifman, Division of Immunology and Allergy, The Hospital for Sick Children, University of Toronto, Toronto, ON

Background: Mutation in the gene IRAK4 (interleukin-1 receptor-associated kinase) is associated with an increased risk of life-threatening Staphylococcus aureus or Streptococcus pneumoniae infection. Objective: To characterize the clinical and immunologic features of the first reported case of twins with IRAK-4 deficiency. Methods: Lymphocyte markers and TCR VBeta distribution were assessed using flow cytometry. Specific antibodies were 
studied using standard methods. Results: Monozygotic twin boys were born to parents who had previously lost a female child to $S$. aureus meningitis at 5 months of age. Between the ages of 10 months and 14 years, twin A developed S. pneumoniae meningitis, septic arthritis, bilateral tonsillar abscesses, brain abscess, and retroperitoneal abscesses. At 15 years, he was found to have an auricular granuloma, a calcified chest nodule on $\mathrm{x}$-ray, and positive blood cultures for Mycobacterium avium. Twin B had pneumococcal meningitis at 2 years of age and supraglottic hemorrhagic necrosis with Pseudomonas aeruginosa at age 5. Antibiotic prophylaxis had little effect on the frequency of infections, but clinical improvement was noted with the addition of intravenous immune globulin. Both interventions were discontinued at adulthood, with no further infections observed. Evaluation of the immune system revealed low numbers of neutrophils and circulating T-cells and a poor antibody response to pneumococcal vaccination. Interestingly, both patients were missing cells expressing TCR VBeta 7.2 chain. Both twins are homozygous and both parents were heterozygous for mutation Q293X in the IRAK-4 gene. Conclusions: IRAK-4 mutation predisposes to life-threatening pyogenic infections in early life. Our patients remain healthy without treatment in the recent decade. The transient nature to the manifestations of IRAK-4 deficiency has yet to be explained. Unique features of our cases include consistent neutropenia, lymphopenia, and infection with M. avium. The scope of the effect of IRAK-4 mutation on immune function may predispose to a broader range of infection than previously identified.

\section{Modulation of Human Mast Cell Activation by Fluticasone and Salmeterol}

L.E. Erdos, R.P. Schleimer, M. Kulka, Northwestern University, Chicago, IL; National Research Council Canada-INH, Charlottetown, PE

Rationale: Glucocorticoids and long-acting beta agonists are effective treatments for asthma. Studying their effects on the human mast cell (MC) will provide a better understanding of the mechanisms of the action of combination therapy in allergic disease. Using a human immortalized MC line (LAD2), we studied the effects of fluticasone propionate (FP) and salmeterol (SM), alone and in combination, on the release of early- and late-phase mediators. Methods: LAD2 cells were treated with FP (100 $\mathrm{nM})$ and $\mathrm{SM}(1 \mu \mathrm{M})$, alone and in combination, at various incubation times and then stimulated with the agonists substance P $(0.5 \mu \mathrm{g} / \mathrm{mL}), \mathrm{C} 3 \mathrm{a}(50 \mathrm{ng} / \mathrm{mL})$, and IgE/anti-
$\operatorname{IgE}(5 \mu \mathrm{g} / \mathrm{mL}, 100 \mu \mathrm{g} / \mathrm{mL})$. Degranulation was measured by the release of $\beta$-hexosaminidase. Cytokine and chemokine expression was measured using microarray analysis, ELISA, and cytometric bead array (CBA) assays. Results: The combination of FP and SM synergistically inhibited degranulation of MCs stimulated with substance P (33\% inhibition compared to control, $n=3, p<.05)$. FP and $\mathrm{SM}$ combined inhibited substance $\mathrm{P}-$ induced release of TNF, MCP-1, and IL-8 (98\%, 99\%, and 92\% inhibition, respectively, $n=4, p<.05)$. Degranulation was inhibited by FP alone but not SM alone when MCs were stimulated with C3a ( $48 \%$ inhibition, $n=3, p<.05)$. FP and SM did not inhibit degranulation when MCs were stimulated with IgE/anti-IgE. Conclusion: Fluticasone and salmeterol synergistically inhibited mediator production by human MCs stimulated with the neuropeptide substance P. This synergistic effect on mast cell signaling may be relevant to the therapeutic benefit of combination therapy in asthma. Research Funding: These studies were supported by a grant from GSK, the Ernest S. Bazely Trust, and grants R01HL068546 and R01HL078860 from the NIH.

\section{The Development of Serum IgE Test Panels for Use by Canadian Primary Care Physicians}

Paul Keith, Susan Wasernman, Milton Gold, Peter Vadas, Adelle Atkinson, Eric Leith, Department of Medicine, McMaster University, Hamilton, ON; Departments of Pediatrics and Medicine, University of Toronto, Toronto, ON

Background: With the rising prevalence of allergy, the public has become increasingly aware of testing for allergy. Primary care physicians are generally the first to assess patients with symptoms suggestive of allergic disease. It was felt that a tool was needed to guide Canadian primary care physicians when they order serum IgE tests for specific symptom profiles. This is especially important in areas of Canada with few practicing allergists. Methods: A group of Canadian allergy specialists met and reviewed symptom profiles and specific IgE test panels previously developed for primary care physicians in Europe, the United Kingdom, and America. Based on clinical experience and published literature, two symptom profiles, one for atopic dermatitis and one for inhalant allergy presenting with rhinitis and/or asthma symptoms, were developed for Canadian primary care physicians. Results: We developed two symptom profiles, one for atopic dermatitis and one for inhalant allergy presenting with rhinitis and/or asthma symptoms. For atopic dermatitis, we suggested specific IgE testing for egg white, cow's milk, fish, wheat, peanut, and soy bean, where these foods are 
relevant to the patient's diet. For rhinitis and/or asthma, we suggested testing for regional tree pollen, regional grass pollen, ragweed, mixed weeds other than ragweed, $D$. pteronyssinus, D. farinae, mould mix, cat, dog, and cockroach, provided that there is a history of relevant exposures. An educational resource document was developed listing the history and physical examination relevant to both disease profiles, as well as guidelines for interpretation of serologic results. Referral to an allergist was strongly recommended if the results were not consistent with the patient's presentation or if the condition did not respond to therapy. Conclusion: Symptom profiles and specific IgE test panels were developed for Canadian primary care practitioners to assist them in choosing appropriate serum IgE tests. Further education of primary care physicians and clear guidelines for interpretation of the results were strongly recommended.

\section{Burden of Illness of Allergic Rhinitis in Canada}

P.K. Keith, M. Desrosiers, S. Waserman, R.R. Schellenberg, Department of Medicine, McMaster University, Hamilton, ON; Department of Medicine, McGill University, University of Montreal, Montreal, QC; Department of Medicine, University of British Columbia, Vancouver, $B C$

Rationale: The objective was to assess the burden of symptoms in Canadian adults with allergic rhinitis (AR). Methods: A cross-sectional, random-digit-dialing telephone survey of 30,987 Canadian households was conducted in July 2006 to identify adult AR patients. After screening 3,671 adults, structured interviews were done with 1,001 respondents (patients diagnosed by a physician as having AR or taking medication for AR). Results: About $45 \%$ of Canadian adults report suffering from nasal symptoms due to allergies unrelated to colds. Less than half (45\%) have been diagnosed by a physician. Half only have seasonal symptoms, with spring and summer being the worst seasons. Of those with AR, 27\% had asthma, $17 \%$ chronic or recurrent sinusitis, and $5 \%$ nasal polyps. More than one-quarter cannot tolerate their symptoms without treatment. Most (83\%) have sought medical attention for their symptoms at one time and onequarter have done so in the past year. The most bothersome symptoms include stuffy nose, runny nose, repeated sneezing, and watering eyes. In their worst month, two-thirds of patients reported having a stuffed nose either daily or several days per week. Almost onequarter reported headaches and sleep loss. One-fifth describe symptoms as poorly controlled or not controlled during the worst month of the year. One-half use only OTC products, $12 \%$ use only prescriptions while onequarter use both. Conclusions: Despite treatment, many Canadians experience allergic rhinitis symptoms that could be better evaluated and controlled. Asthma, sinusitis, and nasal polyposis are common concomitant conditions.

\section{Antibiotic Skin Testing for Children Labeled with Type 1 Hypersensitivity: A Useful Clinical Tool}

Fotini D. Kavadas, Kimberley R. Seaban, Yehuda NofechMozes, Maitham Husain, Elisabeth White, Adelle R. Atkinson,

Division of Immunology and Allergy, Department of Paediatrics, The Hospital for Sick Children, and University of Toronto, Toronto, ON

Background: Children are often unnecessarily labeled as allergic to antibiotics that may be potentially lifesaving. Aside from penicillin, good diagnostic testing has not been available in pediatrics to differentiate between type 1 hypersensitivity and other causes of adverse reactions to antibiotics. The first step would be to determine whether skin testing concentrations are nonirritating and therefore potentially informative of an IgE-mediated reaction. Objectives: (1) To determine whether antibiotic skin testing concentrations used in adults are also nonirritating in children; (2) to describe the potential clinical impact of performing provocative challenges to a wide variety of antibiotics. Methods: A retrospective chart review was done of patients between ages 0 and 18 years who were seen in the Drug Adverse Reaction and Toxicology Clinic over a 2year period with a history of a possible IgE-mediated reaction to various antibiotics other than penicillin. We included patients with either extremely limited antibiotic options or complex medical issues requiring antibiotics. Due to patient safety, we did not perform testing if there was a history of a convincing IgE-mediated or serum sickness-like reaction. Skin testing was done using nonirritating concentrations of the antibiotic in question that have been used in adults. If skin-prick testing was negative, we performed intradermal testing. A provocative challenge was offered if all skin testing was negative. Results: Twenty-three of 28 visits met our inclusion criteria. Four of $23(17 \%)$ could not be skin tested. Of those skin prick tested, all 19 (100\%) had a negative result and 17 of $19(89 \%)$ also had a negative intradermal test. Fifteen of $17(88 \%)$ patients agreed to undergo provocative challenge and 14 of 15 (93\%) were then unlabeled as allergic to the respective antibiotic. Impact: Most of our patients are now able to receive antibiotics that are vital to 
their care and to which they were previously labeled as allergic. Conclusions: We have shown that our antibiotic skin testing concentrations are nonirritating in children. This is a novel tool in pediatrics that may have an important clinical impact in the accurate diagnosis of antibiotic allergies by guiding provocative challenges. In further research, we may consider challenging all patients regardless of the skin test result.

\section{Elevated Histamine Levels Are Associated with Blind Loop Syndrome: A Case Report}

S.C. Paski, C. Kalicinsky, Department of Internal Medicine, University of Manitoba, Winnipeg, $M B$

Jejunoileal bypass is one of the surgical options for morbid obesity and involves creating a blind loop of nonfunctional small bowel to reduce caloric absorption. Blind loop syndrome is a relatively common complication following intestinal bypass surgery. We report a unique case of blind loop syndrome secondary to jejunoileal bypass that presented with symptoms related to elevated histamine. A 58-year-old woman was referred to the Allergy Clinic for assessment of difficult to control adultonset asthma in spite of maximal medical management. She had concomitantly developed recurrent episodes of facial flushing, palpitations, panic, diarrhea, and lightheadedness. Twenty-four-hour urinary histamine levels were markedly elevated at $304 \mu \mathrm{g} / \mathrm{g}$ creatinine (normal $<35$ ). Serum tryptase and serotonin levels were normal. There was no evidence of systemic mastocytosis, carcinoid, pheochromocytoma, cardiac arrhythmia, hyperthyroidism, or occult malignancy. Use of antibiotics was associated with a reduction in histamine production and moderate symptom improvement. Due to the disabling severity of her symptoms, she electively underwent reversal of her jejunoileal bypass and subsequently had complete resolution of her symptoms of histamine excess. Blind loop syndrome is observed in up to $10 \%$ of patients following intestinal bypass surgery. A pronounced increase in histamine excretion has previously been measured in these patients. Histamine is present in appreciable amounts in a normal diet. A low-histamine diet does not reduce histamine excretion in these patients, suggesting that the increase in histamine excretion observed postbypass is a manifestation of the blind loop. The absence of food and pancreatobiliary products in the excluded loop of bowel predisposes it to bacterial colonization with colonic flora. Bacterial colonization of the blind loop has been implicated in several complications post-jejunoileal bypass including arthritis, skin lesions, pneumatosis intestinalis, fever, and liver disease. This case shows that blind loop bacteria are also responsible for elevated histamine production and that elevated histamine is associated with the clinical manifestations of blind loop syndrome.

\section{Effectiveness of Adding Montelukast to Inhaled Corticosteroids in Adult Subjects with Uncontrolled Asthma.}

N. Harvey, R.A. McIvor, S. Coyle, C. Koch, S. Foucart, F. Psaradellis, J.R. Sampalis, J.M. Fitzgerald, on behalf of the Singulair Add-On Study Investigators

Background: Asthma control remains poor despite the widespread utilization of inhaled corticosteroids (ICSs). For patients not controlled with ICSs, the addition of a leukotriene receptor antagonist, such as montelukast, may be beneficial. Methods: An 8-week Canadian multicentre, open-label, prospective cohort study of uncontrolled adult asthma patients treated with ICSs received montelukast sodium $10 \mathrm{mg}$, once daily. Uncontrolled asthma was defined according to the Canadian Asthma Consensus Guidelines. The primary outcome measure was control of asthma as defined by an Asthma Control Questionnaire (ACQ) score $<$ 1.5. Secondary outcomes included a change in ACQ score between baseline and 8 weeks of treatment, patient and physician global satisfaction, and treatment assessments. Results: Of 318 patients enrolled, 303 patients have currently completed 8 weeks of treatment and 284 (94\%) have completed the final assessment. The mean (SD) age was 45.2 (16.2) years, and 207 patients $(68.3 \%)$ were female. There were 189 $(62.4 \%)$ patients with allergic rhinitis. The table describes the efficacy results.

\begin{tabular}{|c|c|c|c|c|c|c|c|}
\hline \multicolumn{4}{|c|}{ ACQ Score Mean (SD) } & \multicolumn{2}{|c|}{ ACQ Score Change from Baseline } & \multicolumn{2}{|c|}{ Subjects with ACQ Score $<1.5$ at 8 wk } \\
\hline $\mathrm{n}$ & Baseline & $\mathrm{n}$ & $8 w k$ & $\mathrm{n}$ & Mean (SD) & $\mathrm{n}$ & $\%$ \\
\hline 303 & $2.11(0.83)$ & 284 & $0.92(0.77)$ & 284 & $-1.14(0.91)^{*}$ & $220(19)^{\dagger}$ & 72.6 \\
\hline
\end{tabular}

${ }^{*} p$ value $<.001$ based on Student's $t$-test for paired samples.

${ }^{\dagger}$ Discontinued patients. 
Physician and patient satisfaction with treatment increased from 41 to $88 \%(p<.001)$ and from 49 to $86 \%(p<.001)$, respectively. For both patients and physicians, $78 \%$ reported overall improvement. Conclusion: Montelukast is effective and well tolerated as add-on therapy for the management of asthma symptoms in patients on ICS with uncontrolled symptoms.

\section{Association between Chronic Urticaria and Autoimmune Thyroid Disease}

Hallaji Zahra, Abdollahi Ali, Barzegari Masoumeh, Tehran University of Medical Sciences, Tehran, Iran

Background: An increase of autoimmune thyroiditis is seen in patients with chronic urticaria, and it has been hypothesized that autoimmunity may be playing a role in the pathogenesis of chronic urticaria. Objectives: In order to examine further the possible relationships between thyroid autoimmunity, thyroid dysfunction, and CU, we have examined thyroid autoantibodies and thyroidstimulating hormone (TSH) levels (an indirect measure of thyroid dysfunction) in chronic urticaria patients. Patients/Methods: We studied 40 patients (30 female and 10 male) with chronic urticaria and 40 age- and sexmatched healthy volunteers. All underwent laboratory tests for thyroid disease with assay of free $\mathrm{T}_{3}$ and $\mathrm{T}_{4}, \mathrm{TSH}$, and antimicrosomal antithyroid antibodies. Results: Twelve patients (11 women and 1 man) found to have antimicrosomal antithyroid antibodies titres ranging from 106 to $960 \mathrm{IU} / \mathrm{mL}$. These antibodies were detected in three control subjects in low titres. The association was statistically significant $(p<.001)$. Four of 12 patients had thyroid dysfunction and the other 8 cases were euthyroid. Conclusions: It seems that thyroid function tests are not enough in chronic urticaria and thyroid antibody evaluation should be carried out in these patients, especially in women.

\section{Do Patients with Asthma Have Their Rescue Inhaler Available for Emergencies?}

K. Gill, C. Sandor, J. Liem, Department of Paediatrics, University of Western Ontario, London, ON; Wayne State University, Detroit, MI; Windsor Allergy and Asthma Education Centre and SWOMEN, University of Western Ontario, Windsor, ON

Rationale: Rescue inhalers are important for the treatment of an acute asthma exacerbation. It is expected that patients with asthma have their rescue inhalers within proximity at all times for emergencies. Objective: To determine whether asthmatics have their rescue inhalers available for emergencies. Methods: Patients in an allergy and asthma clinic were approached and asked if they had asthma. Those who had asthma completed a questionnaire regarding the length of time they had asthma, their medication use, and their inhaler-carrying methods. Results: One hundred twenty patients were approached over 4 clinic days. Thirty-nine (33\%) of those approached had asthma (28 females and 10 males). Eighteen (46\%) of the patients admitted to consistently carrying a rescue inhaler (18 of the females and none of the males, $p<.01$ ). However, only 8 of the patients (all female) were actually carrying an inhaler when asked to have it inspected in clinic. Eleven (28\%) of the patients ( 8 females and 3 males) had severe asthma (defined as greater than two emergency room visits in the past). Of these, only 5 of the female patients carried a rescue inhaler, while none of the male patients carried an inhaler. Conclusions: Patients with asthma do not carry a rescue inhaler at all times to treat an acute exacerbation. More females carried a rescue inhaler compared to males. Severity of asthma is not a predictor of compliance to have a rescue inhaler available for emergencies.

\section{Mast Cell Activation Is Required for the Selective Recruitment of Multiple Dendritic Cell Subpopulations to the Lymph Node}

Wojciech Dawicki, Jean S. Marshall, Dalhousie Inflammation Group, Department of Microbiology and Immunology, Dalhousie University, Halifax, NS

Acquired immune responses are regulated by the nature and activation status of dendritic cell (DC) subpopulations within the lymph node. These DCs activate naive $\mathrm{T}$ cells and help in determining the quantity and quality of the ensuing $\mathrm{T}$ cell responses. The process of $\mathrm{DC}$ activation may be modified in allergic diseases and may enhance inappropriate $\mathrm{T}$ cell responses. We have examined the role mast cell activation plays in the recruitment of several DC subpopulations into the lymph node in response to both IgE/antigen and peptidoglycan from $S$. aureus (PGN). Local activation of murine skin mast cells with specific IgE followed by antigen led to significant $(p$ $<.05)$ increases in the numbers of $\mathrm{CD}^{+} \mathrm{DCs}, \mathrm{CD} 11 \mathrm{~b}^{+}$ DCs, and plasmacytoid DCs (pDCs) in the lymph node draining the site of activation. By analyzing mast celldeficient mice as well as those having local mast cell reconstitution, we observed that mast cells are required for the recruitment of $\mathrm{CD}^{+}$and $\mathrm{pDCs}$ but not $\mathrm{CD} 11 \mathrm{~b}^{+} \mathrm{DCs}$ into the lymph node in response to intradermal admin- 
istration of PGN. TNF-deficient mice had an equivalent level of lymph node activation, both in terms of DC recruitment and increases in total lymph node cellularity in response to PGN, suggesting that TNF is not critical to this process. In contrast, IL-6-deficient mice had significantly $(p<.01)$ impaired recruitment of $\mathrm{CD} 11 \mathrm{~b}^{+}$DCs following activation with PGN. These data show that mast cell activation can selectively promote the accumulation of DC subpopulations in the lymph node in response to either PGN or IgE/antigen challenge and highlight the potential role of mast cells in the modulation of acquired immune responses relevant to allergic disease. This work is supported by the Canadian Institutes of Health (CIHR). W. Dawicki is supported by a fellowship from the CIHR, Canadian Lung Association, and GlaxoSmithKline.

\section{Eosinophil Infiltration in Oral Squamous Cancer: Role of prostaglandin $D_{2}$}

Francis Davoine, Adrian Sim, Charlie Tang, Sibina Fisher, Lakshmi Puttagunta, Tim McGaw, Donald Yu, Lisa Cameron, Darryl J. Adamko, Redwan Moqbel, Pulmonary Research Group, Campus-Saint-Jean, Department of Laboratory Medicine and Pathology, Department of Medical Microbiology and Immunology, Department of Dentistry, Department of Paediatrics, University of Alberta, Edmonton, $A B$

Background: Eosinophils are an important inflammatory leukocyte infiltrating oral squamous cell carcinomas (OSCs). A number of studies suggested that eosinophils may provide good prognosis in OSC; however, this area is controversial. Conflicting evidence exists regarding the exact role eosinophils in tumour regression, as well as factors leading to their recruitment and prognostic value. Prostaglandins are known to be secreted by oral carcinomas and may, therefore, be involved in promoting eosinophil infiltration. Objectives: To investigate the mechanisms underlying recruitment of human eosinophils to sites of selective tumour growth. In particular, we investigated the role of prostaglandins and the role they potentially play in antineoplastic activity of eosinophils. Methods: Luna and MBP staining for eosinophils in surgically resected primary OSC was performed and correlated with tumour staging/grade and clinical outcome. Blood eosinophils were purified from eosinophilic subjects by immunomagnetic negative selection. The antineoplastic effect of eosinophils was determined by coculture assay with an OSC cell line, SCC-9. Eosinophil degranulation was evaluated by peroxidase (EPO) release colorimetric assay (OPD). Eosinophil chemotaxis toward
OSC was measured by infiltration and migration through an artificial basement membrane, Matrigel. Results: Eosinophil infiltration was observed around the tumour mass in a preliminary cohort of 21 subjects with OSC. Eosinophil infiltration appeared to be more prominent in lower grade/stage OSC. Deposition of $\mathrm{MBP}^{+}$granules was also observed in the the vicinity of the tumour mass. We observed approximately $40 \%$ growth inhibition of SCC-9 during coculture for 72 hours with eosinophils. This growth inhibition correlated with EPO activity. SCC-9 induced strong transmigration of eosinophils $(30 \pm 5 \%, n$ $=14, p<.0001)$ comparable to eotaxin control $(38 \pm$ $6 \%)$. The $\mathrm{PGD}_{2}$ synthase inhibitor HQL-79 limited eosinophil migration toward SCC-9. SCC-9 cells also increased the expression of $\mathrm{PGD}_{2}$ receptor (CRTH2) at the surface of eosinophils. Conclusion: Our results suggest that eosinophils are selectively recruited by OSC-secreted $\mathrm{PGD}_{2}$ and possessed strong growth-inhibiting activity in the surrounding of the tumour mass. These properties may be implicated in the positive prognosis associated with eosinophilic infiltration in OSC. These observations suggest that modulating eosinophilia in certain types of cancers may have potential as adjuvant immunotherapy.

\section{Relationship between Metabolic Syndrome, Asthma, and Airway Hyperresponsiveness}

N. Saurek-Aleksandrovska, A.L. Kozyrskyj, R. Rabbani, A.B. Becker, E.A.C. Sellers, Manitoba Institute for Child Health; Department of Community Health Sciences, Faculty of Pharmacy, Department of Pediatrics and Child Health, Department of Pediatrics and Endocrinology, University of Manitoba, Winnipeg, $M B$

Objective: To determine the prevalence of metabolic syndrome among asthmatic and nonasthmatic children in prepuberty and the association between metabolic syndrome, asthma, and airway hyperresponsiveness (AHR). Methods: Data were obtained from the SAGE case-control study of the 1995 Manitoba birth cohort. Metabolic syndrome was established if children had three of five elements (central obesity, high fasting blood glucose, high blood pressure, low HDL, and high triglycerides). Fasting blood was obtained for glucose, triglycerides, and HDL. Waist circumference and blood pressure were measured three times and averaged. Asthma was diagnosed by a pediatric allergist and AHR was measured using the methacholine challenge test. The association between metabolic syndrome and asthma was expressed as the odds ratio (OR) and 95\% confidence interval (CI). Results: Among 559 children (346 nonasthmatic and 189 asthmatic 
children) at 10 to 11 years of age, the prevalence of metabolic syndrome was $4.3 \%$. It was more common in children with asthma (5.3\%) compared to those without (3.9\%), but the association was not significant ( OR = 1.31, 95\% CI 0.57-3.00). The OR between metabolic syndrome and prevalent asthma in girls was higher but also not significant $(\mathrm{OR}=1.64,95 \%$ CI $0.36-7.52)$. No association was also observed between metabolic syndrome and AHR or asthma and AHR in all children or in boys and girls separately. Conclusion: There was no association between metabolic syndrome at age 10 to 11 and prevalent asthma or AHR, but we are in the process of recruiting children for the third wave of the SAGE study and soon will have results on the association between metabolic syndrome and incident asthma at age 12 years.

\section{Overweight at 8 to 10 Years Old Trends Toward a Higher Prevalence of Asthma among Girls but not Boys}

J.L.P. Protudjer, A.L. Kozyrskyj, R. Rabbani, A.B. Becker, University of Manitoba, Winnipeg, MB; Department of Applied Health Sciences, National Training Program in Allergy and Asthma, Manitoba Institute of Child Health, Faculty of Pharmacy, Department of Community Health Sciences, Department of Pediatrics and Child Health, Section of Clinical Immunology

Rationale: Asthma and obesity have increased dramatically in recent years. While generally viewed as associated conditions, recent studies suggest the possibility of obesity as a causal factor in the development of asthma in females from puberty onward. As part of a longitudinal study, we considered the possibility that overweight antedates asthma in 11- to 12-year-old girls but not 11- to 12year-old boys. Methods: Children enrolled in the SAGE 1995 Manitoba Birth Cohort were assessed by a pediatric allergist for asthma and had anthropometric measurements taken at ages 8 to 10 years and 11 to 12 years. Heights and weights were converted to age- and genderappropriate body mass indices and classified as normal weight $(<85$ th percentile $)$ or overweight $(\geq 85$ th percentile). The likelihood of asthma at age 11 to 12 was ascertained for overweight status at age 8 to 10 by the odds ratio (OR) and $95 \%$ confidence interval (CI). Results: Nine of the 20 children (4/13 boys; 5/7 girls) who were overweight at age 8 to 9 were assessed as having asthma at age 11 to 12 . Considering both genders together, overweight at age 8 to 9 did not predict asthma at age 11 to 12 (OR 1.17; 95\% CI 0.42-3.29; NS). Nor was this relationship significant among boys (OR 0.41; 95\% CI
0.10-1.67; NS). However, girls who were overweight at age 8 to 10 were strongly trended toward an increased prevalence of asthma at age 11 to 12 (OR 5.56; 95\% CI $0.90-34.25 ; p=.052)$. Conclusions: In this preliminary analysis, girls who were overweight at age 8 to 9 tended to have a greater likelihood of developing asthma at age 11 to 12 than boys who were overweight at age 8 to 9. Data collection is ongoing; we predict that this trend will become significant as more children are assessed.

\section{Anaphylaxis to Chicken and Turkey: A Case Report}

L. Connors, G. Sussman, alhousie University, Halifax, NSa; University of Toronto, Toronto, ON

Background: Food allergy to avian meats, including chicken and turkey, is not commonly described. The birdegg syndrome has been described to include allergy to eggs as well as avian meats. There are few case reports that describe allergy to avian meats without egg allergy. Methods: A case of chicken and turkey allergy is presented. Clinical presentation, skin-prick testing results, and RAST results are presented. Results: A 27-year-old female had six episodes of mild to moderate anaphylaxis between December 2005 and November 2006. Initially, sesame was felt to be the culprit allergen; however, anaphylaxis recurred without sesame, and when the patient ingested sesame, no reaction occurred. Further questioning revealed chicken was ingested prior to the reactions for the first five episodes and turkey prior to the final episode. Skin-prick testing was positive for chicken and turkey but negative for egg and sesame. RAST was positive for chicken and turkey and negative for sesame. Conclusion: This case describes IgE-mediated immediate hypersensitivity to chicken with cross-reactivity to turkey. The patient was not egg allergic and was not tested for other avian meats as she does not plan to ingest them in the future. Previous case reports have shown allergy to chicken and turkey as well as other avian meats. This case outlines the importance of a detailed history in determining possible causative agents of anaphylaxis.

\section{Aseptic Meningitis following Intravenous Immunoglobulin Administration in a Series of Patients with Chronic Urticaria}

Sari M. Herman-Kideckel, Gordon Sussman, Stephen Betschel, Division of Clinical Immunology and Allergy, Department of Medicine, University of Toronto, Toronto, ON

Background: Chronic idiopathic urticaria (CIU) is a debilitating condition that is often exceedingly challenging to manage. Intravenous immunoglobulin (IVIG) has been 
used for its treatment in patients unresponsive to conventional therapies. Aseptic meningitis is a recognized but uncommon adverse reaction reported after IVIG infusion. Purpose: To report a series of patients who developed aseptic meningitis following IVIG administration. We review the published literature examining aseptic meningitis with the use of IVIG and propose mechanisms for its development in patients with CIU. Methods: We report three patients with a history of CIU refractory to conventional therapy who were treated with IVIG. Results: A 3-day course of IVIG at $1 \mathrm{~g} / \mathrm{kg} / \mathrm{d}$ was initiated in three patients. Within 48 hours, all patients developed severe headache, meningismus, nausea, vomiting, photophobia, and malaise. Physical examination revealed nuchal rigidity, and a lumbar puncture was performed in all patients. Analysis of the cerebrospinal fluid demonstrated a pleocytosis with a predominance of neutrophils without positive culture and confirmed the diagnosis of aseptic meningitis. Urticaria improved in all patients after the IVIG treatment. Retreatment was initiated in one patient for recurrence of CIU and was tolerated without side effects. Conclusions: IVIG has been used for the treatment of CIU in patients unresponsive to conventional therapies. Although IVIG therapy is generally safe, aseptic meningitis is a potentially serious complication. Patients with CIU receiving IVIG may be at higher risk of developing aseptic meningitis. Patients should be informed of the possible adverse effects related to IVIG therapy.

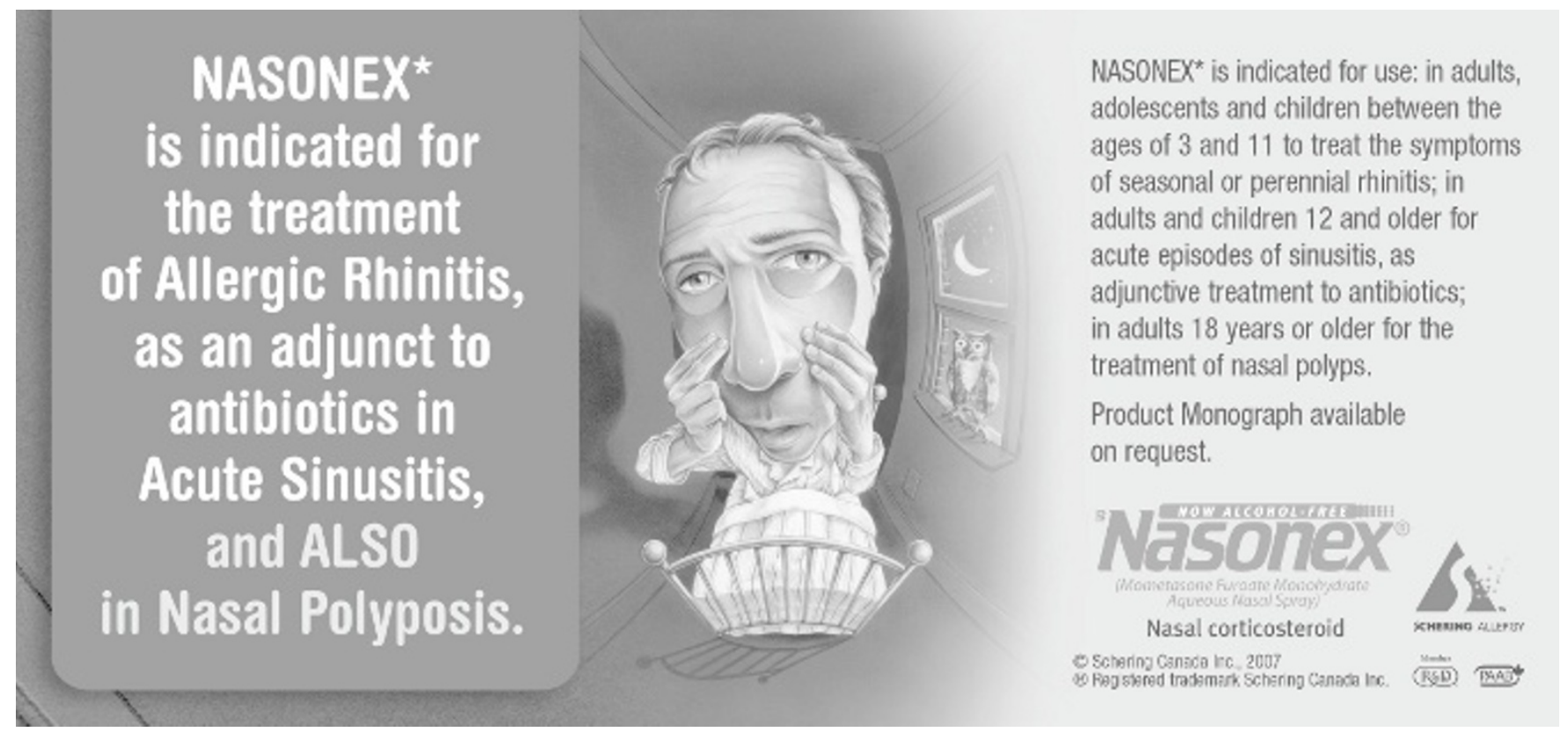

